\title{
Decellularized aortic conduits: could their cryopreservation affect post-implantation outcomes? A morpho-functional study on porcine homografts
}

\author{
Michele Gallo $^{1} \cdot$ Antonella Bonetti $^{2} \cdot$ Helen Poser $^{3} \cdot$ Filippo Naso $^{1} \cdot$ Tomaso Bottio $^{1}$. \\ Roberto Bianco $^{1} \cdot$ Adolfo Paolin $^{5} \cdot$ Paolo Franci $^{3} \cdot$ Roberto Busetto $^{3}$. \\ Anna Chiara Frigo ${ }^{6} \cdot$ Edward Buratto $^{7} \cdot$ Michele Spina $^{4} \cdot$ Maurizio Marchini $^{2}$. \\ Fulvia Ortolani $^{2} \cdot$ Laura Iop $^{1,8} \cdot$ Gino Gerosa $^{1,8}$
}

Received: 30 November 2015 / Accepted: 15 April 2016

(C) Springer Japan 2016

\begin{abstract}
Decellularized porcine aortic valve conduits (AVCs) implanted in a Vietnamese Pig (VP) experimental animal model were matched against decellularized and then cryopreserved AVCs to assess the effect of cryopreservation on graft hemodynamic performance and propensity to in vivo repopulation by host's cells. VPs $(n=12)$ underwent right ventricular outflow tract substitution using AVC allografts and were studied for 15-month follow-up. VPs were randomized into two groups, receiving AVCs treated with decellularization alone ( $\mathrm{D} ; n=6)$ or decellularization/ cryopreservation (DC; $n=6$ ), respectively. Serial echocardiography was carried out to follow up hemodynamic function. All explanted AVCs were processed for light and electron microscopy. No signs of dilatation, progressive stenosis, regurgitation, and macroscopic calcification were echocardiographically observed in both D and DC groups.
\end{abstract}

M. Gallo and A. Bonetti contributed equally to this work.

F. Ortolani, L. Iop and G. Gerosa are equally responsible for the concept and design of the study.

Michele Gallo

michelegallo@hotmail.co.uk

Gino Gerosa

gino.gerosa@unipd.it

1 Division of Cardiac Surgery, Department of Cardiac, Thoracic and Vascular Sciences, University of Padua, Via Giustiniani 2, 35128 Padua, Italy

2 Department of Experimental and Clinical Medicine, University of Udine, Udine, Italy

3 Department of Animal Medicine, Productions and Health, University of Padua, Legnaro, Italy
Explanted D grafts exhibited near-normal features, whereas the presence of calcification, inflammatory infiltrates, and disarray of elastic lamellae occurred in some DC grafts. In the unaltered regions of AVCs from both groups, almost complete re-endothelialization was observed for both valve cusps and aorta walls. In addition, side-by-side repopulation by recipient's fibroblasts, myofibroblasts, and smooth muscle cells was paralleled by ongoing tissue remodeling, as revealed by the ultrastructural identification of typical canals of collagen fibrillogenesis and elastogenesis-related features. Incipient neo-vascularization and re-innervation of medial and adventitial tunicae of grafted aortic walls were also detected for both D and DC groups. Cryopreservation did not affect post-implantation AVC hemodynamic behavior and was topically propensive to cell repopulation and tissue renewal, although graft deterioration including calcification was present in several areas. Thus, these preliminary data provide essential information on feasibility of decellularization and cryopreservation coupling in the perspective of treatment optimization and subsequent clinical

4 Department of Biomedical Sciences, University of Padua, Padua, Italy

5 Tissue Bank of Veneto Region, Treviso Regional Hospital, Treviso, Italy

6 Department of Cardiac, Thoracic and Vascular Sciences, Biostatistics, Epidemiology and Public Health Unit, University of Padua, Padua, Italy

7 Department of Cardiothoracic Surgery, Royal Children's Hospital, Murdoch Children's Research Institute, University of Melbourne, Melbourne, Australia

8 Cardiovascular Regenerative Medicine, Venetian Institute of Molecular Medicine (VIMM), Biomedical Campus Pietro d'Abano, Padua, Italy 
trials using similarly treated human allografts as innovative heart valve substitutes.

Keywords Bioengineering $\cdot$ Heart valve .

Decellularization - Cryopreservation - Echocardiography · Preclinical model

\section{Introduction}

Valvular heart disease represents a major public health problem responsible for significant morbidity and mortality worldwide. Valve replacement surgery is the leading therapeutic option, with approximately 300,000 valve prostheses required per year, worldwide [1]. The European Homograft Bank has recently reported a total of 4516 allograft valve implantations during the last 20 years for the repair or reconstruction of diseased ventricular outflow and inflow tracts (1391 aortic, 2620 pulmonary, and 48 mitral) [2]. Nevertheless, available allograft valves in cryopreserved stocks are insufficient to meet clinical demand. Long-term failure rate of allogeneic valve conduits is similar to that described for glutaraldehyde-treated xenografts [3]. Clinical studies have demonstrated that allograft failure could be at least partly the result of cellular and humoral immune responses to human leukocyte antigens (HLA), which are present in implanted allografts [4]. Although good preservation of the extracellular matrix (ECM) has been reported for cryopreserved allografts [5, 6], damage to collagen and/ or elastin was shown to occur during the cryopreservation/ thawing process $[7,8]$ as result of crystal ice formation and/or proteinase release by non-viable cells. These injured tissue elements may exacerbate the calcification process [9], which is primed by hydroxyapatite nucleation on dying valve interstitial cells and their debris in both in vivo [10] and in vitro [11] experimental models.

Previous in vivo investigation indicated that decellularized pulmonary valves implanted in the ovine right ventricular outflow tract (RVOT) exhibited a lower incidence of cusp and wall calcification than cryopreserved native pulmonary valves [12]. Furthermore, the hemodynamic performance of the decellularized pulmonary valves was comparable to the cryopreserved counterparts, exhibiting significantly lower pressure gradients compared with current glutaraldehyde-treated bioprostheses (Medtronic, Inc, Freestyle Bioprosthesis; Medtronic, Minneapolis, MN) [13]. To avoid the problems caused by valvular tissue treatment with glutaraldehyde, physical crosslinking procedures were employed such as dye mediated photo-oxidation [14].

The combination of osmotic shock, cell membrane emulsification with Triton X-100/Sodium Cholate (TRICOL), and nucleic acid digestion with aspecific endonucleases has proven to be a very promising decellularization procedure. In fact, it provides a suitable preservation of the texture of native valve interstitium and hemodynamic performance [15-17], with concurrent removal of the xenoantigen galactose- $\alpha-1,3$-galactose (alpha-gal) [18]. In addition, TRICOL-treated porcine aortic valve conduits (AVCs) were shown to exhibit physiological performance for up to 15 months after implantation in Vietnamese Pigs (VPs) as well as permissivity to repopulation by recipient's cells and tissue remodeling capability, as revealed by ex vivo morphological analyses [19, 20].

Currently, the creation of innovative bioengineered heart valves represents a promising research field. Coupling decellularization and cryopreservation procedures would be an ideal strategy for an off-the-shelf, self-regenerating, functional valve prostheses, allowing both long-term storage and ready availability.

In the perspective of future clinical trials, this preliminary study focuses on the impact of combined TRICOL decellularization and subsequent standard cryopreservation on AVC allografts using the validated VP animal model, at least showing propensity to cell repopulation and tissue renewal.

\section{Materials and methods}

\section{Isolation, decellularization, and cryopreservation of aortic allografts}

Porcine hearts $(n=12)$ were harvested from young common breeding piglets (3-4 months old) at a local abattoir and stored in sterile cold saline solution with a full complement of antimicrobial agents (penicillin $100 \mathrm{U} / \mathrm{mL}$, streptomycin $100 \mathrm{mg} / \mathrm{mL}$, and amphotericin B $250 \mu \mathrm{g} / \mathrm{mL}$ ) until further processing. AVCs were isolated by aseptic excision performed according to an established tissue harvest procedure [19], with a warm ischemic time less than $1 \mathrm{~h}$. TRICOL decellularization of 12 AVCs was carried out as previously described [15]. Briefly, aortic homografts were treated with a combination of osmotic shock and a Triton X100 plus sodium cholate (TRICOL) detergent solution. An endonuclease treatment (Benzonase, Merck, Darmstadt, Germany) was applied to remove remaining nuclear fragments. Before being frozen, decellularized AVCs were immersed in a cryopreservant solution composed of RPMI 1640 with $0.3 \mathrm{~g} / \mathrm{L}$ of L-Glutamine (Sigma-Aldrich, Saint Louis, MO, USA) and containing $10 \%$ dimethylsulfoxide (Sigma-Aldrich, Saint Louis, MO, USA) and $10 \%$ of $20 \%$ human albumin (Kedrion Biopharma, Inc., Fort Lee, NJ, USA) as cryoprotective agents. AVCs were sterilely maintained in low-temperature-resistant polypropylene package and cryocooled in a liquid nitrogen computerised freezer (ICECUBE 1860, Icemachinedepot, Fayetteville, NC, 
USA) with controlled temperature lowering $\left(-1{ }^{\circ} \mathrm{C} / \mathrm{min}\right.$ until $-140{ }^{\circ} \mathrm{C}$ ). Subsequently, storage was performed in the accredited "Tissue Bank of Veneto Region" (Regional Authorization number 3948, 15 December 2000) under controlled conditions between -140 and $-185{ }^{\circ} \mathrm{C}$ in a liquid nitrogen vapor tank.

\section{VP chronic implant model}

The Italian Ministry of Health authorized the University of Padua to use VPs for the experimental purposes of this study (27/08 C16 project), conforming to D. Lgs. n.116 (art. 12; January 27, 1992). All animal experiments and surgical procedures were performed in compliance with ISO 10993-1, ISO 10993-2, and UNI EN ISO 5840 standards. VPs $(n=12)$ were randomized into group $\mathrm{D}(n=6)$, consisting in VPs subjected to RVOT substitution with allogeneic TRICOL-decellularized AVCs, and group DC $(n=6)$, consisting in VPs subjected to RVOT substitution with TRICOL-decellularized and cryopreserved AVCs. AVCs from both groups were implanted in 12-month-old VPs $(54.3 \pm 2.5$ and $69.0 \pm 3.7 \mathrm{~kg}$ for females and males, respectively) using beating heart cardiopulmonary bypass. Surgical procedure and postoperative management were performed as previously described [19-21].

\section{Echocardiography}

Echocardiographic evaluation [22] was performed under general anesthesia preoperatively at 15 days and 1, 3, 6, 12, 15 months after surgery. Transthoracic 2D echocardiographic analyses were carried out using an ultrasound device (Zonare ${ }^{\mathrm{TM}}$ Zone Ultra, Mountain View, USA) equipped with a phased array sector transducer (1-4 MHz). Transesophageal 3D live echocardiography was performed using a iE33 xMATRIX (Philips, Eindhoven, The Netherlands). Valve function was assessed evaluating pulmonary stenosis (peak transvalvular gradient; PTG), pulmonary regurgitation $($ absent $=0$, trivial $=1$ st degree, mild $=2$ nd degree, moderate $=3$ rd degree, and severe $=4$ th degree , and right ventricular function (secondary tricuspid regurgitation).

\section{Explantation of AVC conduits}

AVCs were explanted under general anesthesia 15 months after implantation, and animals were euthanized with Tanax [20]. Both D and DC AVCs were excised under aseptic conditions, examined macroscopically, and documented photographically.

\section{Histology}

Samples of explanted AVCs were fixed with phosphatebuffered $4 \%$ paraformaldehyde and cut to obtain specimens comprising the valve cusp and a segment of the adjacent aortic wall as well as the anastomosis between the donor's aortic wall and the recipient's pulmonary one. Specimens were then paraffin-embedded, cut into $4-\mu \mathrm{m}$-thick sections, and stained with: (1) hematoxylin and eosin (H\&E) (2) Masson's trichrome, (3) Weigert's method for elastic fiber selective staining, (4) alcian blue dissolved in sodium acetate buffer containing $0.05 \mathrm{M}$ magnesium chloride for total glycosaminoglycan (GAG) visualization, and (5) von Kossa method for selective visualization of calcium-binding sites. Observations and photographic recordings were made using a Zeiss AxioImager photomicroscope (Carl Zeiss, Oberkochen, Germany).

\section{Immunohistochemistry}

Histological sections were deparaffinized and pre-treated with a phosphate-buffered saline (PBS) solution containing $0.1 \%$ Triton X-100 for $10 \mathrm{~min}$ and a PBS solution containing $3 \%$ hydrogen peroxide for $5 \mathrm{~min}$. Sections were then incubated with PBS solutions containing (1) $3 \%$ normal donkey serum for $40 \mathrm{~min}$, (2) 1:1500 rabbit anti-PGP 9.5 polyclonal antibody (UltraClone Limited, Rossiters Farm House Wellow, Isle of Wight, England) or 1:100 rabbit anti-von Willebrand factor (Dako, Glostrup, Denmark) for 90 min at r.t. in a humidified chamber, and (3) 1:600 donkey anti-rabbit peroxidase-conjugated secondary antibody (Jackson ImmunoResearch Laboratories, West Grove, PA) for $30 \mathrm{~min}$. Peroxidase activity was revealed by incubation with diaminobenzidine chromogen (BioGenex, San Ramon, CA) for $3 \mathrm{~min}$. Finally, immunoreacted sections were faintly counterstained with haematoxylin, dehydrated in graded ethanols, cleared with xylene, and mounted with Eukitt ${ }^{\circledR}$. As negative controls, primary antibody was replaced with normal donkey serum. Observations and photographic recordings were performed as above.

\section{Transmission electron microscopy}

Samples of explanted AVCs were fixed with phosphatebuffered $2.5 \%$ glutaraldehyde and $2.5 \%$ formaldehyde and cut to obtain valve cusp and aortic wall specimens which were: (1) post-fixed with $2 \%$ osmium tetraoxide (Agar Scientific, Essex, England), (2) dehydrated in graded ethanols, and (3) embedded in Araldite/Epon (Agar Scientific, Essex, England). Ultrathin sections were collected on 
formvar-coated $2 \times 1$-mm-slot copper grids and contrasted with uranyl acetate and lead citrate. Observations and photographic records were made using a Philips CM12/ STEM electron microscope (Philips, Eindhoven, The Netherlands).

\section{Statistical analysis}

All data are reported as mean and standard deviation. Differences between groups were analyzed using Mann-Whitney $U$ test. Intra-group differences during follow-up were evaluated using Wilcoxon signed-rank test. A $p$ value of 0.05 was considered to be statistically significant [23]. For statistical analyses, SAS 9.1 statistical software package (SAS Institute Inc., Cary, NC, USA) was used.

\section{Results}

\section{Operative results}

Both D and DC AVCs showed adequate mechanical properties during surgical implantation and weaning from extracorporeal circulation. Early surgical complications, such as excessive bleeding with cardiac tamponade, graft displacement, and sternal dehiscence, did not occur, demonstrating that VPs are eligible for the surgical technique. One animal in D group showed a progressive valvular stenosis with severe right ventricular failure due to massive endocarditis and thrombosis in the graft, so it was excluded from the study. In DC group, one animal died due to ventricular fibrillation after surgery and necropsy revealed native endocarditis in the tricuspid valve. Iatrogenic complications due to AVC damage during surgical anastomosis affected another animal. These two distinct animals were also excluded from the study.

\section{Echocardiographic assessment}

AVCs from both D and DC groups showed no echocardiographic structural deterioration, evident signs of cusp thickening or reduction of cusp mobility or microbial vegetations during the 15 months of follow-up. During followup, PTGs in D and DC groups 15 days after surgery were $27.8 \pm 5.8$ and $36 \pm 16.6 \mathrm{mmHg}$, respectively. The gradients remained stable over time $(p=0.1224)$, with PTGs at month 15 measuring $31.8 \pm 13.4$ and $20.5 \pm 4.1 \mathrm{mmHg}$, respectively, without significant difference between the two groups $(p=0.2509)$. However, these values were significantly higher $(p<0.001)$ than the preoperative recordings $(2.66 \pm 0.9$ and $2.75 \pm 1.2 \mathrm{mmHg}$ for D and DC group, respectively) (Fig. 1a). A significant increase (trivial $\backslash$ mild regurgitation) in pulmonary insufficiency $(p=0.0015)$ was noted in both D and DC groups comparing preoperative data (trivial regurgitation) with those recorded 15 months after graft implantation (trivial $\backslash$ mild regurgitation), although these values remained unchanged over the postoperative time $(p=0.0880)$, with no significant difference between the two groups $(p=0.8561)$ (Fig. 1b). For both groups, the right ventricular function was preserved and there was no significant secondary tricuspid insufficiency (Fig. 1c). Hyperechogenic areas were assessed near the proximal and distal sutures, as expected.

\section{Macroscopic examination of explanted allografts}

Macroscopically, no recognizable signs of tissue alterations (graft rupture, cusp thickening, fibrin deposits, gross calcification, and microbial vegetation) were found for D allografts (Fig. 2), except for the presence of two punctate opacified regions affecting one valve cusp in one of the five grafts. Conversely, DC allografts exhibited apparently near-normal regions coexisting with others showing alterations such as opacization, stiffening, and thickening of valve cusps and/or aorta walls. These injuries were heterogeneously sized and randomly distributed, involving either valve cusps or aortic walls, as noted for two grafts, and both valve cusps and aortic walls, as noted for the other two grafts.

\section{Light microscopy}

Histologically, both positive features and negative ones were observed, with worse patterns being mainly shown by DC samples. Since the aim of this study is to provide improvement in processing of cadaver-derived aortic valve substitutes, only pictures of DC samples will be illustrated.

In general, unaltered regions in both D and DC allograft cusps were characterized by a native-like distribution of collagen fibers, elastic lamellae, and GAG component (Fig. 3a, c, g, i). Cusp cell repopulation was also apparent.

Namely, the valve cusps appeared to be covered by a monolayer of flattened cells (Fig. 3a, c, g, i), showing immunopositivity for endothelial cell marker von Willebrand factor (Fig. 3e).

Moreover, a side-by-side distribution of irregularly or spindle-shaped cells was observed throughout the cusp interstitium (Fig. 3a, c, g, i). Monolayered flattened cells were found to cover also the aortic wall luminal surfaces (Fig. 3b, d), exhibiting immunopositivity for endothelial cell marker von Willebrand factor (Fig. 3f). Host cells were also found to repopulate tunica media and, at greater extent, tunica adventitia (Fig. 3b, d, h), in which ECM components showed an apparently unaltered array of collagen (Fig. 3b, h) and elastin components (Fig. 3d). In addition, evident revascularization occurred in the outer tunica media and the 


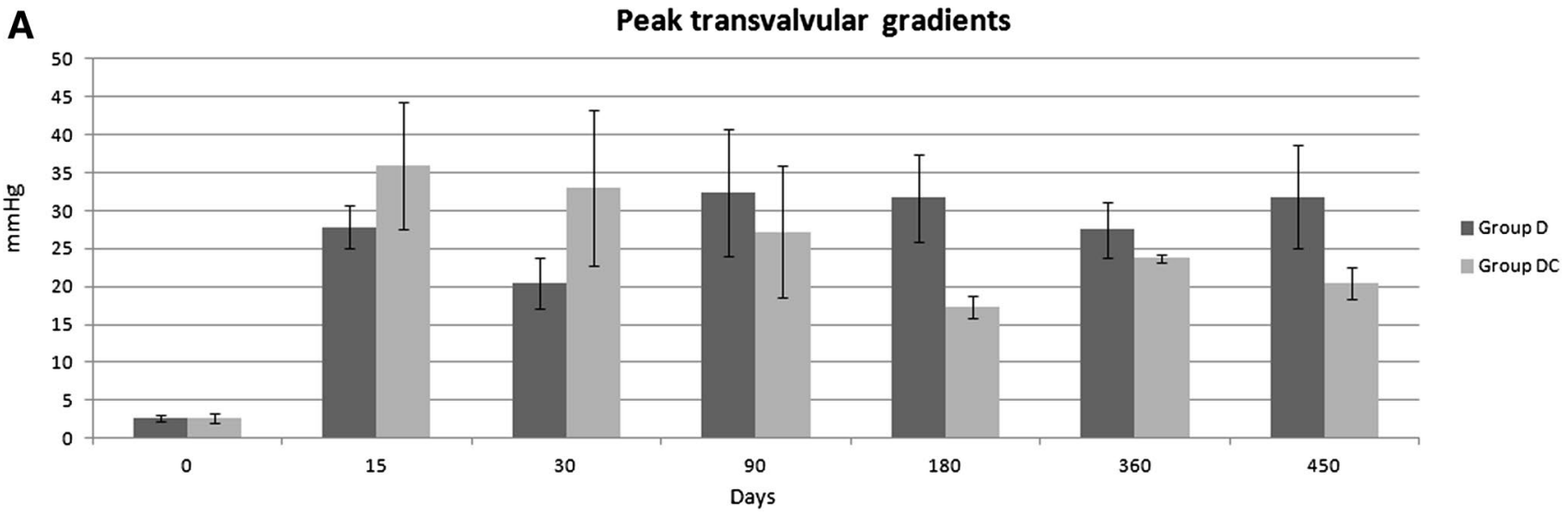

B

Pulmonary Regurgitation

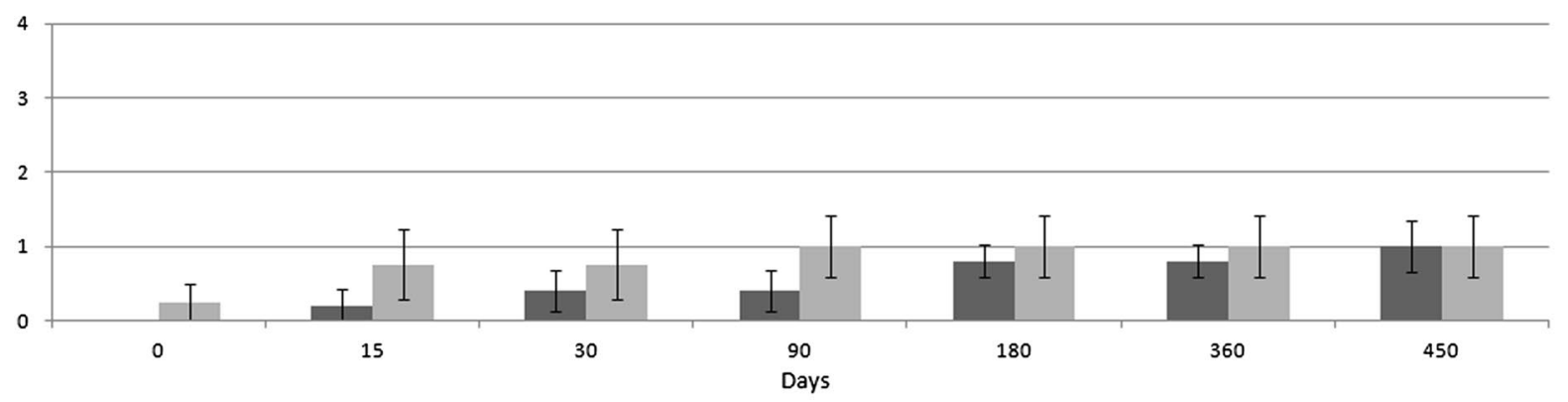

\section{c}

Tricuspid Regurgitation

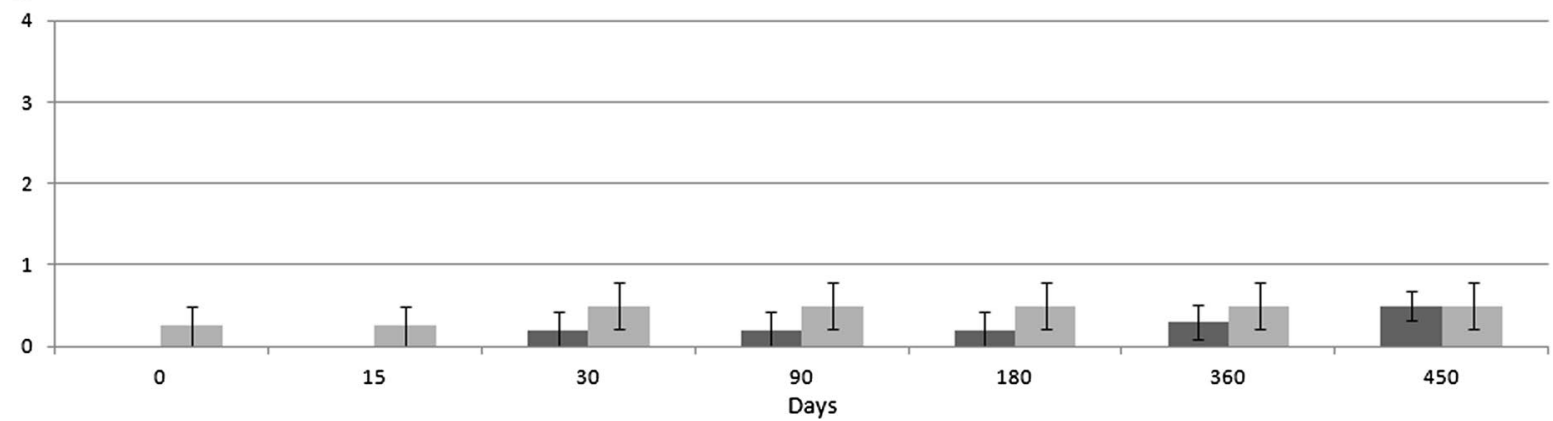

Fig. 1 Echocardiographic assessment of $\mathrm{D}(n=5)$ allografts compared with DC $(n=4)$ counterparts. Peak transvalvular gradients were significantly higher than the preoperative values, although they remained stable over time. No significant differences were appreciable between the two groups (a). Pulmonary regurgitation was trivial $\backslash$ mild after implantation without worsening during the follow-up in both D and DC groups (b). Secondary tricuspid regurgitation was absent/trivial in both groups $($ c) $($ absent $=0$; trivial $=1$ st degree; mild $=2$ nd degree; moderate $=3$ rd degree; severe $=4$ th degree) . Data are reported as mean value (histograms) and standard deviation (bold line)
Fig. 2 Macroscopic features of explanted allografts. Opacification of aortic wall was observed after explantation in DC group, while cusps were translucent and surface did not present macroscopic alterations (a). Aorta wall of D group showed a normal appearance without fibrin deposits (b)
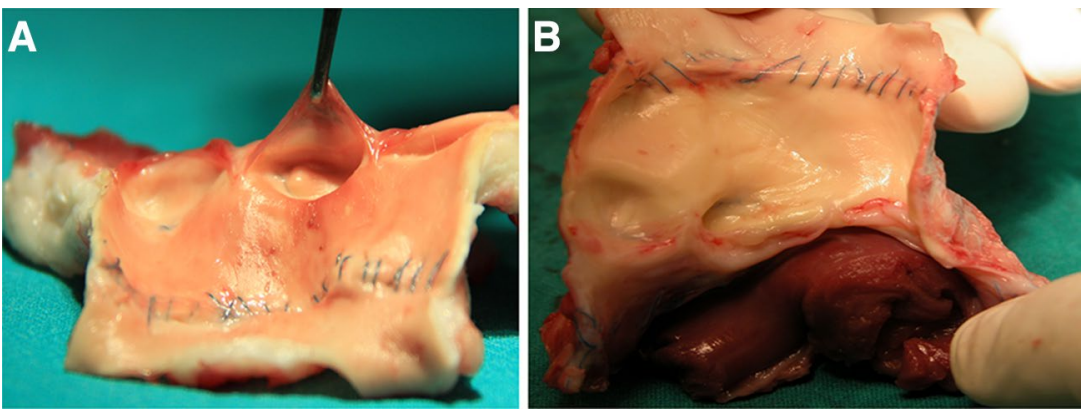
Fig. 3 Histological and immunohistochemical analyses of DC allografts. AVC cusps showing re-endothelialization of both their aspects $(\mathbf{a}, \mathbf{c}, \mathbf{g}, \mathbf{i}$, arrowheads) by cells showing immunopositivity for von Willebrand factor (e) as well as sideby-side cell repopulation (a, $\mathbf{c}, \mathbf{g}, \mathbf{i})$, flanked by native-like distribution of collagen fibers $(\mathbf{a}, \mathbf{g})$, elastin lamellae (c), and glycosaminoglycan component (i). AVC walls showing focal re-endothelialization of luminal surfaces (arrowheads in $\mathbf{b}, \mathbf{d}$ ) by intimal cells immunopositive for von Willebrand factor (f), besides repopulation of tunica media (b, d), containing apparently unaltered elastic laminae (d), and tunica adventitia (h, j), containing both newly formed blood vessels (h, arrows) and PGP-9.5-immunopositive nerve fibers (j, arrows). Bar $0.5 \mathrm{~mm}$ $(\mathbf{a}-\mathbf{d}, \mathbf{g}-\mathbf{i}) ; 120 \mu \mathrm{m}(\mathbf{e}, \mathbf{f})$; $0.4 \mathrm{~mm}(\mathbf{j})$
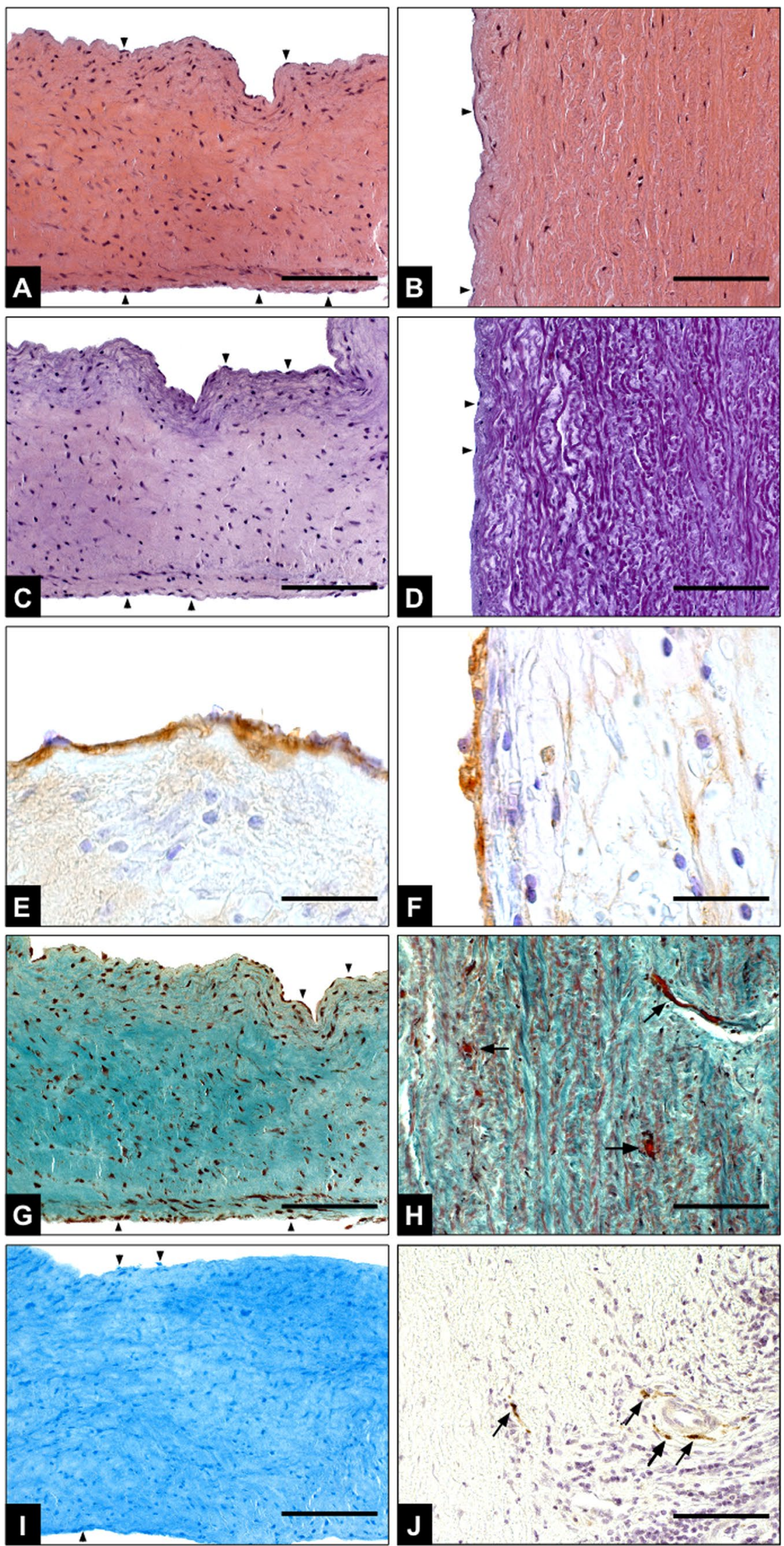
entire tunica adventitia (Fig. 3h), the latter also demonstrating incipient re-innervation, as revealed by immunopositivity of thin nerve fibers for PGP 9.5 neural marker (Fig. 3j). However, important tissue alterations, such as presence of fibrin deposits, inflammatory infiltrates, calcific foci, scarce GAG component, and fragmented elastin fibers, were also encountered in both valve cusps and aorta walls, with these adverse outcomes occurring more frequently in the DC group than the $\mathrm{D}$ group.

\section{AVC ultrastructure}

Ultrastructural analysis of unaltered AVC samples from both D and DC groups better revealed the presence of ongoing tissue regeneration. Namely, endothelial-like flat cells were found to cover both aspects of grafted valve cusps, being (1) arranged as monolayers, (2) interconnected by immature tight junctions, and (3) underlined by thin basal laminae (Fig. 4a). In the valve cusp interstitium, several repopulating cells exhibited fibroblast features (Fig. 4b), while other cells were rich in intracytoplasmatic myofilaments and interconnected by immature cell junctions, resembling myofibroblasts, the irregularly shaped ones (Fig. 4c), and smooth muscle cells, the oblong ones (Fig. 4d). In addition, clear features of ongoing ECM neosynthesis were apparent, such as (1) presence of collagen fibrils having smaller diameters than in mature fibrils in adult valve cusps (Fig. 4c-f), (2) abundance of the characteristic canal-shaped cell indentations involved in collagen fibrillogenesis (Fig. 4e), and (3) prominent juxtacellular distribution of small immature elastin fibers, i.e., elaunin fibers associated with abundant fibrillin microfibrils (Fig. 4f). Similar cell repopulation patterns and associated ECM neo-synthesis were apparent in the aortic walls (Fig. 5a-e). In addition, newly formed intramural blood vessels were encountered (Fig. 5f), with luminal surfaces covered by joined endothelium-like cells underlined by continuous basal laminae, some of which exhibited multiple arrangement as often occurring during angiogenic processes (Fig. 5g).

\section{Discussion}

The ideal bioengineered AVC would be a fully compatible graft, with the potential to grow with the patient, remodel, and self-regenerate. From our previous experience [19-21], spontaneous tissue-guided regeneration of acellular scaffolds from native heart valves proved to be an appealing technique for a straight-forward and functional clinical application. Previous studies utilize as animal model the juvenile sheep and more recently also the elderly sheep [24-26]. In this context, the allogeneic VP model has been shown to be a suitable experimental system for in vivo, long-term validation of valve prostheses, since it produces coagulation and inflammatory responses, as in transplanted patients. In the present study, the same VP animal model was used to match the performance of TRICOL-decellularized valve allografts against that of TRICOL-decellularized valve allografts subsequentially subjected to cryopreservation. Since cryopreservation is mandatory to preserve human allogeneic valves for use in valve replacement, this study may be conceived as an anticipation of a possible human clinical trial. Both D and DC aortic allografts used in this study were obtained from breeding piglets and implanted in the pulmonary position of VPs to anticipate the potential outcomes deriving from their clinical use as RVOT substitutes. Decellularized valvular scaffolds were found to undergo progressive cell repopulation, acting as functional substitutes at least for 15 months, without showing any dilatation or failure. Although a limitation of this study was the relatively small number of enrolled animals, statistical analysis indicated that both D and DC grafts did not show significant time-related increasing in PTGs after implantation. These results are in line with other reports regarding biological valvular scaffolds, for which the gradients remained constant during the entire period of followup [24-27]. However, PTGs were significantly higher than the preoperative gradients and the short-term nature of this event precludes the genesis of a pathological stenosis. It is likely that PTG modification may depend on mechanical factors, such as anatomy of aortic root, patient-prosthesis mismatch, or pressure recovery, and biological factors, such as infection, coagulation, inflammation, cell repopulation, and ECM synthesis [28, 29]. Of interest, an in vitro study demonstrated that TRICOL-treated valves showed lower gradients than in controls (non-decellularized, native valves) [17]. Conversely, right ventricular function was preserved, as indicated by absence of significant secondary tricuspid insufficiency for both groups. Both D and DC grafts also showed trivial/mild regurgitation after surgery, which persisted for entire follow-up, without increasing. These results correlate with our previous in vitro studies reporting a prolonged phase of cusp coaptation during diastole concerning TRICOL-treated valve prostheses [17]. The adopted decellularization treatment, therefore, is likely to make the cusp more flexible, facilitating the trivial/ mild regurgitation observed in vivo. However, comparing this parameter with that related to autografts repositioned in orthotopic position, no significant differences were observed [19]. Conversely, decellularizing treatments using trypsin/EDTA were seen to be more aggressive and induce structural damage, causing moderate valvular regurgitation soon after implantation [30, 31].

Since cryopreservation is employed to allow ready availability and long-term storage of valve prostheses, the 

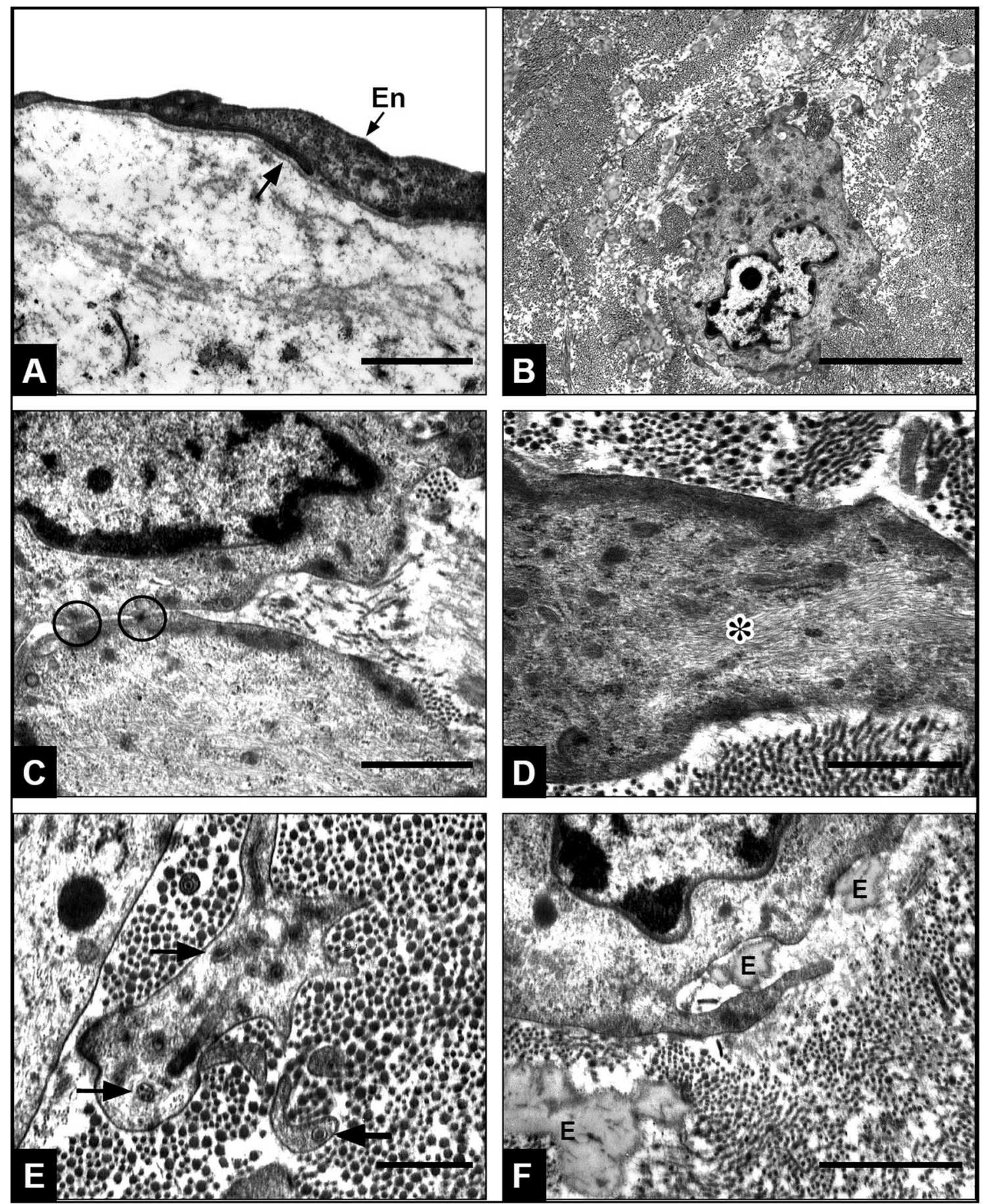

Fig. 4 Ultrastructure of aortic valve cusps from DC allografts. AVC cusps lined by surfacing endothelial-like cells (a En = endothelium) with a thin basal lamina (arrow); a fibroblast-like cell populating a native-like interstitium (b); two myofibroblast-like cells (c) interconnected by cell junctions (circles); native-like smooth muscle cells (d)

present study tested the in vivo performance of TRICOLdecellularized and then cryopreserved valve allografts. Notably, the obtained results revealed that cryopreservation does not affect the functional performance of DC filled with actin myofilaments (asterisk); presence of characteristic native-like canals of collagen fibrillogenesis (arrows in e) and neosynthesis of elastin fibers (f $\mathrm{E}=$ elaunin fibers). $\operatorname{Bar} 1 \mu \mathrm{m}(\mathbf{a}, \mathbf{c}, \mathbf{d}, \mathbf{f})$; $5 \mu \mathrm{m}(\mathbf{b}) ; 0.5 \mu \mathrm{m}(\mathbf{e})$

allografts. Moreover, despite the possibility of cryopreservation-dependent graft alterations [7-9], AVCs from DC group were found to be permissive to cell repopulation, as revealed by the identification of recipient's cells colonizing 

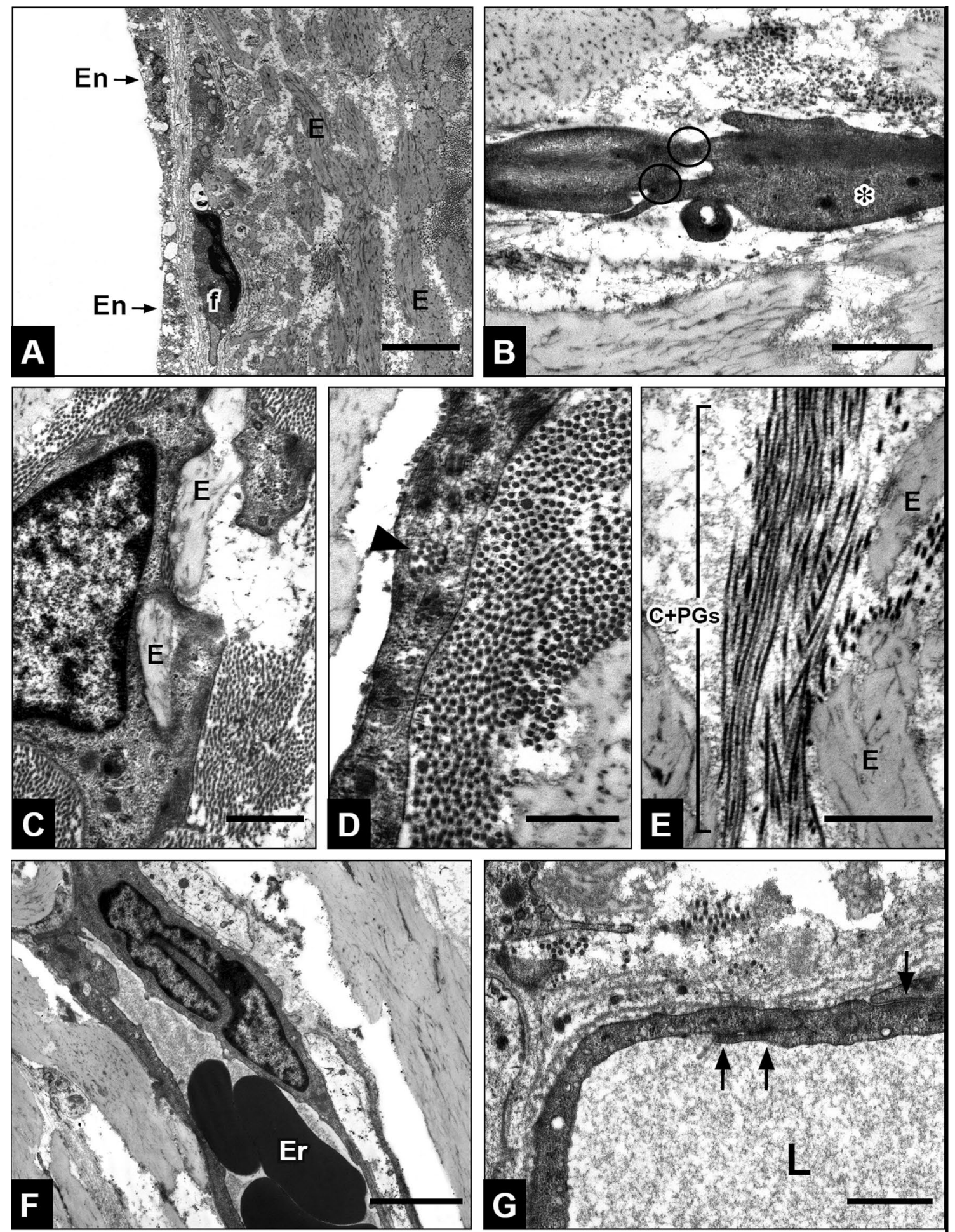

Fig. 5 Ultrastructure of aortic valve walls from DC allografts. AVC walls showing endothelial-like cells covering the aorta luminal surface $(\mathbf{a} \mathrm{En}=$ endothelium; $\mathrm{f}=$ sub-endothelial fibroblast; $\mathrm{E}=$ elastin fibers). Tunica media showing native-like smooth muscle cells (b) interconnected by cell junctions (circles) and rich in actin myofilaments (asterisk); myofibroblast-like cells involved in elastin fiber neo-synthesis (c E = elaunin fibers); fibroblast-like cells (d) showing canals of collagen fibril neo-synthesis (arrowhead). Media extracel-

lular matrix (e) exhibiting native-like arrayed collagen fibrils, periodically interconnected by orthogonal small proteoglycans (C + PGs), and mature elastin fibers (e) with the presence of regenerated blood vessels (f) containing mature erythrocytes $(\mathrm{Er})$. Blood vessel $(\mathbf{g})$ lined by interconnected endothelial-like cells (arrows; $\mathrm{L}=$ lumen) underlined by a multiple basal lamina. $\operatorname{Bar} 2.5 \mu \mathrm{m}(\mathbf{a}, \mathbf{f}) ; 1 \mu \mathrm{m}$ (b, c, e, g); $0.5 \mu \mathrm{m}(\mathbf{d})$ 
the aortic graft, as well as ECM neo-synthesis, demonstrated by the presence of (1) typical canals of collagen fibrillogenesis, (2) abundant small-diameter collagen fibrils, and (3) elaunin and immature elastic fibers, as observed for $\mathrm{D}$ grafts. Of note, such tissue regeneration features were accompanied by re-vascularization and re-innervation of the tunica adventitia and the outer tunica media of grafted aorta walls, key events for valve tissue renewal and functionality. On the other hand, re-endothelialization of valve cusps and aorta wall luminal surfaces was seen to be more complete for grafted AVCs from D group. It is likely that cryopreservation affects the integrity of native endothelium basal lamina, compromising its preservation after TRICOL treatment alone [19] and exposing DC grafts to fibrin deposition and/or infiltration by inflammatory cells.

Morphologically, DC allografts exhibited calcification spots in both valve cusps and aorta walls. Betchel et al. reported a high frequency of calcification (26\%) in SynerGraft $^{\circledR}$ allografts (Cryolife), as revealed by CT scan postoperatively after 10-month follow-up. However, no significant change in valvular function was echocardiographically detected [32]. The Cryolife use as cryoprotectant solution a Dulbecco's Modified Eagle's Medium (DMEM) containing $10 \% \mathrm{v} / \mathrm{v}$ fetal bovine serum and $10 \% \mathrm{v} / \mathrm{v}$ dimethyl sulfoxide [6]. A survey conducted in 24 international heart valve banks show numerous differences on heart valve processing techniques used in term of sterile culture media, cryoprotectant reagent, antibiotic/antimycotic cocktail, controlled rate freezing for cryopreservation, and storage of allografts at ultralow temperatures [33]. These findings suggest that ECM damage induced by cryopreservation and thawing is mainly responsible for calcification, since decellularization treatment preserves allografts from cell death-associated tissue mineralization, which commonly affects native human homografts [4, 9-11]. To avoid cryopreservation, Cebotari et al. reported the use of fresh decellularized valves that are stored up to 3 weeks at $+4{ }^{\circ} \mathrm{C}$ before implantation. However, this technique requires that patients await graft availability and are admitted to the hospital at short notice [34]. Indeed, the impact of cryopreservation on the scaffold texture is paramount for the long-term durability of decellularized heart valves. Schenke-Layland reported that collagen and elastin architecture was specifically disrupted in response to the cryopreservation/thaw cycling [8] and proposed a novel heart valve preservation method which ensures ECM preservation avoiding ice formation and tissue-glass cracking [35]. On the contrary, Gerson et al. indicated that conventional cryopreservation of human heart valve allografts does not affect collagen and elastin structural integrity [6]. Narine et al. investigated the effects of cryopreservation on the structural and biochemical properties of decellularized porcine aortic valves, which was found to not significantly alter collagen and uronic acid content of aortic cusp matrices. However, histological and ultrastructural analyses showed extensive disruption to affect collagen fibrils as well as inter-fibrillar small proteoglycans [36].

It is well known that the extent of freezing/thawing damage depends upon the amount of free water in the ECM and its propensity to crystallization. The major role of cryoprotectants, when used at high concentrations, seems to be the induction of vitrification, i.e., extracellular glass formation. Compared to traditional cryopreservation, vitrification promotes non-crystalline or amorphous water solidification rather than crystallization, limiting the amount of ice crystal formation [33]. Currently, a promising cryoprotectant is VS83, consisting in a combination of $4.65 \mathrm{M}$ dimethylsulfoxide, $4.65 \mathrm{M}$ formamide, and 3.3 M 1,2-propanediol [37].

On the whole, the present results indicate that cryopreservation mitigates some of the favorable outcomes provided by TRICOL decellularization treatment. However, it is worth noting that neither allograft hemodynamic performance, as revealed by the functional comparison, nor their propensity to appropriate cell repopulation and associated tissue renewal, as shown histologically and ultrastructurally, were compromised.

Future investigations will combine TRICOL decellularizing treatment and ice-free cryopreservation [34] for in vivo evaluation of hemodynamic performance, propensity to cell repopulation, and tissue remodeling capability of bioengineered valve substitutes in long-term follow-up.

\section{Compliance with Ethical Standards}

Funding This study was funded by the RSF 286/08 Regione Veneto Grant and Azione Biotech III.

Conflict of interest The authors do not have any conflicts to disclose. No competing financial interests exist.

Ethical approval All applicable international, national, and/or institutional guidelines for the care and use of animals were followed.

\section{References}

1. Biancari F, Barbanti M, Santarpino G, Deste W, Tamburino C, Gulino S, Immè S, Di Simone E, Todaro D, Pollari F, Fischlein T, Kasama K, Meuris B, Dalén M, Sartipy U, Svenarud P, Lahtinen J, Heikkinen J, Juvonen T, Gatti G, Pappalardo A, Mignosa C, Rubino AS (2016) Immediate outcome after sutureless versus transcatheter aortic valve replacement. Heart Vessels 31:427-433

2. Jashari R, Goffin Y, Van Hoeck B, Vanderkelen A, du Verger A, Fan Y, Holovska V, Fagu A, Brahy O (2010) Belgian and European experience with the European Homograft Bank (EHB) cryopreserved allograft valves assessment of a 20 year activity. Acta Chir Belg 110(3):280-290

3. Kobayashi J, Kawashima Y, Matsuda H, Nakano S, Kasugai T, Tokuan Y (1990) Pathological findings of the aortic homograft in 
a patient with tetralogy of Fallot twenty years after implantation. Heart Vessels 5(2):98-101

4. Breinholt JP, Hawkins JA, Lambert LM, Fuller TC, Profaizer T, Shaddy R (2000) A prospective analysis of the immunogenicity of cryopreserved non valved allografts used in pediatric heart surgery. Circulation 7 102(19 Suppl 3):III179-III182

5. Lang SJ, Giordano MS, Cardon-Cardo C, Summers BD, StaianoCoico L, Hajjar DP (1994) Biochemical and cellular characterization of cardiac valve tissue after cryopreservation or antibiotic preservation. J Thorac Cardiovasc Surg 108:63-67

6. Gerson CJ, Heacox AE (2009) Retained structural integrity of collagen and elastin within cryopreserved human heart valve tissue as detected by two-photon laser scanning confocal microscopy. Cryobiology 59(2):171-179

7. Brockbank KG, Lightfoot FG, Song YC, Taylor MJ (2000) Interstitial ice formation in cryopreserved homografts: a possible cause of tissue deterioration and calcification in vivo. J Heart Valve Dis 9:200-206

8. Schenke-Layland K, Madershahian N, Riemann I, Starcher B, Halbhuber KJ, König K, Stock UA (2006) Impact of cryopreservation on extracellular matrix structures of heart valve leaflets. Ann Thorac Surg 81:918-926

9. Mitchell RN, Jonas RA, Schoen FJ (1998) Pathology of explanted cryopreserved allograft heart valves: comparison with aortic valves from orthotopic heart transplants. J Thorac Cardiovasc Surg 115:118-127

10. Ortolani F, Bonetti A, Tubaro F, Petrelli L, Contin M, Nori SL, Spina M, Marchini M (2007) Ultrastructural characterization of calcification onset and progression in subdermally implanted aortic valves. Histochemical and spectrometric data. Histol Histopathol 22(3):261-272

11. Bonetti A, Della Mora A, Contin M, Tubaro F, Marchini M, Ortolani F (2012) Ultrastructural and spectrophotometric study on the effects of putative triggers on aortic valve interstitial cells in vitro models simulating metastatic calcification. Anat Rec Hoboken 295(7):1117-1127

12. Affonso da Costa FD, Dohmen PM, Lopes SV, Lacerda G, Pohl F, Vilani R, Affonso Da Costa MB, Vieira ED, Yoschi S, Konertz W, Affonso da Costa I (2004) Comparison of cryopreserved homografts and decellularized porcine heterografts implanted in sheep. Artif Organs 28:366-370

13. Quinn RW, Hilbert SL, Bert AA, Drake BW, Bustamante JA, Fenton JE, Moriarty SJ, Neighbors SL, Lofland GK, Hopkins RA (2011) Performance and morphology of decellularized pulmonary valves implanted in juvenile sheep. Ann Thorac Surg 92(1):131-137

14. Verbrugghe P, Meuris B, Flameng W, Herijgers P (2009) Reconstruction of atrioventricular valves with photo-oxidized bovine pericardium. Interact Cardiovasc Thorac Surg 9(5):775-779

15. Spina M, Ortolani F, ElMesslemani A, Gandaglia A, Bujan J, Garcia-Honduvilla N, Vesely I, Gerosa G, Casarotto D, Petrelli L, Marchini M (2003) Isolation of intact aortic valve scaffolds for heart-valve bioprostheses: extracellular matrix structure, prevention from calcification, and cell repopulation features. J Biomed Mater Res A 67(4):1338-1350

16. Iop L, Renier V, Naso F, Piccoli M, Bonetti A, Gandaglia A, Pozzobon M, Paolin A, Ortolani F, Marchini M, Spina M, De Coppi P, Sartore S, Gerosa G (2009) The influence of heart valve leaflet matrix characteristics on the interaction between human mesenchymal stem cells and decellularized scaffolds. Biomaterials 30:4104-4116

17. Bottio T, Tarzia V, Dal Lin C, Buratto E, Rizzoli G, Spina M, Gandaglia A, Naso F, Gerosa G (2010) The changing hydrodynamic performance of the decellularized intact porcine aortic root: considerations on in vitro testing. J Heart Valve Dis 19:485-491
18. Naso F, Gandaglia A, Iop L, Spina M, Gerosa G (2011) First quantitative assay of alpha-Gal in soft tissues: presence and distribution of the epitope before and after cell removal from xenogeneic heart valves. Acta Biomater 7:1728-1734

19. Gallo M, Naso F, Poser H, Rossi A, Franci P, Bianco R, Micciolo M, Zanella F, Cucchini U, Aresu L, Buratto E, Busetto R, Spina M, Gandaglia A, Gerosa G (2012) Physiological performance of a detergent decellularized heart valve implanted for 15 months in Vietnamese pigs: surgical procedure, follow-up, and explant inspection. Artif Organs 36(6):E138-E150

20. Iop L, Bonetti A, Naso F, Rizzo S, Cagnin S, Bianco R, Dal Lin C, Martini P, Poser H, Franci P, Lanfranchi G, Busetto R, Spina M, Basso C, Marchini M, Gandaglia A, Ortolani F, Gerosa G (2014) Decellularized allogeneic heart valves demonstrate selfregeneration potential after a long-term preclinical evaluation. PLoS One 9(6):e99593

21. Gallo M, Bianco R, Bottio T, Naso F, Franci P, Zanella F, Perona G, Busetto R, Spina M, Gandaglia A, Gerosa G (2013) Tissueengineered heart valves: intra-operative protocol. J Cardiovasc Transl Res 6(4):660-661

22. Rossi A, Faggiano P, Amado AE, Cicoira M, Bonapace S, Franceschini L, Dini FL, Ghio S, Agricola E, Temporelli PL, Vassanelli C (2014) Mitral and aortic valve sclerosis/calcification and carotid atherosclerosis: results from 1065 patients. Heart Vessels 29(6):776-783

23. Brunner E, Langer F (2000) Nonparametric analysis of ordered categorical data in designs with longitudinal observations and small sample sizes. Biom J 42(6):663-675

24. Theodoridis K, Tudorache I, Calistru A, Cebotari S, Meyer T, Sarikouch S, Bara C, Brehm R, Haverich A, Hilfiker A (2015) Successful matrix guided tissue regeneration of decellularized pulmonary heart valve allografts in elderly sheep. Biomaterials $52: 221-228$

25. Baraki H, Tudorache I, Braun M, Hffler K, Grler A, Lichtenberg A, Bara C, Calistru A, Brandes G, Hewicker-Trautwein M, Hilfiker A, Haverich A, Cebotari S (2009) Orthotopic replacement of the aortic valve with decellularized allograft in a sheep model. Biomaterials 30(31):6240-6246

26. Hopkins RA, Jones AL, Wolfinbarger L, Moore MA, Bert A, Lofland GK (2009) Decellularization reduces calcification while improving both durability and 1-year functional results of pulmonary homograft valves in juvenile sheep. $\mathrm{J}$ Thorac Cardiovasc Surg 137(4):907-913

27. Vincentelli A, Wautot F, Juthier F, Fouquet O, Corseaux D, Marechaux S, Le Tourneau T, Fabre O, Susen S, Van Belle E, Mouquet F, Decoene C, Prat A, Jude B (2007) In vivo autologous recellularization of a tissue-engineered heart valve: are bone marrow mesenchymal stem cells the best candidates? J Thorac Cardiovasc Surg 134(2):424-432

28. Kasimir M, Weigel G, Sharma J, Rieder E, Seebacher G, Wolner E, Simon P (2005) The decellularized porcine heart valve matrix in tissue engineering: platelet adhesion and activation. Thromb Haemost 94(3):562-567

29. Rieder E, Seebacher G, Kasimir M, Eva Eichmair, Winter B, Dekan B, Wolner E, Simon P, Weigel G (2005) Tissue engineering of heart valves: decellularized porcine and human valve scaffolds differ importantly in residual potential to attract monocytic cells. Circulation 111(21):2792-2797

30. Cebotari S, Lichtenberg A, Tudorache I, Hilfiker A, Mertsching H, Leyh R, Breymann T, Kallenbach K, Maniuc L, Batrinac A, Repin O, Maliga O, Ciubotaru A, Haverich A (2006) Clinical application of tissue engineered human heart valves using autologous progenitor cells. Circulation 114(1 Suppl):I132-I137

31. Huang SD, Liu XH, Bai CG, Lu FL, Yuan Y, Gong DJ, Xu ZY (2007) Synergistic effect of fibronectin and hepatocyte growth factor on stable cell-matrix adhesion, re-endothelialization, and 
reconstitution in developing tissue-engineered heart valves. Heart Vessels 22(2):116-122

32. Bechtel JF, Gellissen J, Erasmi AW, Petersen M, Hiob A, Stierle U, Sievers HH (2005) Mid-term findings on echocardiography and computed tomography after RVOT-reconstruction: comparison of decellularized (SynerGraft) and conventional allografts. Eur J Cardiothorac Surg 27(3):410-415

33. Heng WL, Albrecht H, Chiappini P, Lim YP, Manning L (2013) International heart valve bank survey: a review of processing practices and activity outcomes. J Transplant 2013:163150

34. Cebotari S, Tudorache I, Ciubotaru A, Boethig D, Sarikouch S, Goerler A, Lichtenberg A, Cheptanaru E, Barnaciuc S, Cazacu A, Maliga O, Repin O, Maniuc L, Breymann T, Haverich A (2011) Use of fresh decellularized allografts for pulmonary valve replacement may reduce the reoperation rate in children and young adults: early report. Circulation 13 124(11 Suppl):S115-S123
35. Lisy M, Pennecke J, Brockbank KG, Fritze O, Schleicher M, Schenke-Layland K, Kaulitz R, Riemann I, Weber CN, Braun J, Mueller KE, Fend F, ScheunertT Gruber AD, Albes JM, Huber AJ, Stock UA (2010) The performance of ice-free cryopreserved heart valve allografts in an orthotopic pulmonary sheep model. Biomaterials 31(20):5306-5311

36. Narine K, Ing EC, Cornelissen M, Desomer F, Beele H, Vanlangenhove L, Smet SD, Nooten GV (2006) Readily available porcine aortic valve matrices for use in tissue valve engineering. Is cryopreservation an option? Cryobiology 53(2):169-181

37. Brockbank KG, Wright GJ, Yao H, Greene ED, Chen ZZ, Schenke-Layland K (2011) Allogeneic heart valve storage above the glass transition at $-80^{\circ} \mathrm{C}$. Ann Thorac Surg 91(6):1829-1835 